\title{
Diagnóstico del estado de operación comercial y opinión sobre los efectos económicos y financieros en las empresas como consecuencia de la pandemia COVID-19
}

\author{
Mauricio Azálgara Bedoya \\ Universidad Internacional Iberoamericana, Calle 15 num. 36, entre 10 y 12, IMI III, Campeche, México \\ Enviado el 14 de noviembre del 2021. Aceptado el 8 de enero del 2022.
}

DOI: https://doi.org/10.33017/RevECIPeru2021.0009/

\section{Resumen}

Habiéndose tenido acceso a datos abiertos procedentes de la Encuesta de Opinión sobre el Impacto del COVID19 en las empresas llevada a cabo por el Instituto Nacional de Estadística e Informática (INEI), se tiene que la presente investigación constituye un soporte informativo acerca del impacto del COVID-19 en el entorno empresarial peruano durante el 2 do trimestre de 2020. Se divulga que el impacto es poco favorable para el desarrollo empresarial ya que se evidencia la inoperatividad u operatividad parcial de las empresas, la disminución de ventas, el funcionamiento con un nivel de capacidad instalada menor al regular, la tenencia de menor cantidad de trabajadores, la tenencia de problemas financieros, y la poca accesibilidad a la asistencia gubernamental para la reactivación.

Descriptores: Empresas peruanas, COVID-19, opinión empresarial, operación comercial, estado empresarial

\section{Abstract}

Having had access to open data from the Opinion Survey on the Impact of COVID-19 on companies carried out by the National Institute of Statistics and Informatics (INEI), the present research constitutes informative support about the impact of COVID-19 in the Peruvian business environment during the 2nd quarter of 2020. It is disclosed that the impact is unfavourable for business development since it is evidenced the following issues: the inoperative or partial operation of the companies, the decrease in sales, the operation with a lower level of installed capacity, the participation of fewer workers, the presence of financial problems, and the little accessibility to government assistance for reactivation.

Keywords: Peruvian companies, COVID-19, business opinion, business operation, business status

\section{Introducción}

Durante el año 2020 se promulgó el Decreto Supremo No 044-2020-PCM que declara Estado de Emergencia Nacional por las graves circunstancias que afectan la vida de la Nación a consecuencia del brote del COVID-19 [1]; las disposiciones del decreto se han ido prorrogando de mes en mes mientras que dura la pandemia. Se tiene que las disposiciones del Decreto Supremo No 044-2020-
PCM afectan la actividad económica por lo que se promulga el Decreto Supremo $N^{\circ}$ 080-2020-PCM con la finalidad de comenzar la recuperación social y económica dando prioridad a la transición hacia una reanudación de actividades que incluya precauciones y medidas de protección necesarias para prevenir los contagios y minimizar el riesgo de un repunte de la enfermedad [2]. 
Se considera que la presente investigación está alineada en su contenido con el diagnóstico empresarial provisto por ciertas instituciones nacionales que proveen cierta información acerca del entorno empresarial peruano vinculado con la pandemia del COVID-19. Por un lado, la Dirección de Promoción Minera [3] afirma que el producto bruto interno $(\mathrm{PBI})$ se contrajo en $11 \%$ en el año 2020, además, se aprecia que la recuperación económica nacional mejoró por la reanudación de actividades económicas en el 2 do semestre de 2020. Por otro lado, se tiene a la Sociedad Nacional de Industrias [4] que divulga que cerca del $92 \%$ de empresas no operan regularmente a abril de 2020 .

Dado el contexto anterior, la presente investigación aprovecha estadísticamente los datos del Instituto Nacional de Estadística e Informática (INEI) respecto de la encuesta de opinión empresarial peruana sobre el impacto del COVID-19 que obtiene información para conocer la opinión sobre los efectos económicos y financieros en las empresas por efecto de la pandemia del COVID-19 [5]; cabe mencionar que a la fecha no se ha publicado documento oficial del Instituto Nacional de Estadística e Informática que refiere al uso de estos datos. Adicionalmente, la presente investigación analiza tanto el entorno empresarial peruano impactado por la pandemia del COVID-19 como ciertas políticas públicas y privadas que se han aplicado durante la pandemia.

\subsection{Fundamentos del problema}

\subsubsection{El planteamiento del problema}

Las entidades públicas y privadas tienen necesidad entre otros de tener conocimiento del impacto del COVID-19 en las empresas peruanas, además de la opinión empresarial respecto de la pandemia. La necesidad expuesta tiene su fundamento en lo siguiente: (1) se requiere conocer el estado de los sectores económicos durante la pandemia; (2) se desconocen algunos aspectos de la temática empresarial durante la pandemia en el Perú; (3) se desconoce el impacto del COVID-19 en las empresas peruanas; (4) se desconoce la efectividad de los programas o medidas de incentivo y/o reactivación brindadas por el gobierno peruano.

Junto con la necesidad de conocimiento expuesta anteriormente se aprecia en el entorno peruano una dificultad para acceder a la información empresarial dado que la pandemia constituye para las empresas un evento del cual no se preparó ningún tipo de plan de contingencia lo cual se tradujo en un cuello de botella en el flujo de información empresarial. A lo anterior, se suma la realidad de contar con una tenencia de recursos escasos e insuficientes de parte de varias instituciones para llevar a cabo una encuesta empresarial. No obstante, se cuenta con datos abiertos recolectados por el Instituto Nacional de Estadística e Informática (INEI) acerca del entorno empresarial durante la pandemia COVID19; los datos proceden de la encuesta de opinión sobre el impacto del COVID-19 en las empresas de una muestra de 2,354 empresas que se ejecutó en el mes de julio de 2020 teniendo como periodo de referencia el segundo trimestre de 2020. A la fecha, no se cuenta con un documento oficial que refiera a tales datos por lo que se considera a tales datos de tal encuesta como no procesados o en proceso de procesarlos para efectos del presente documento.

\subsubsection{La formulación del problema}

¿Cuál es el impacto de la pandemia del COVID-19 en las empresas peruanas durante el segundo trimestre de 2020?

\subsubsection{La sistematización del problema}

¿En qué estado se encuentra la operación comercial de las empresas peruanas a consecuencia de la pandemia del COVID-19 durante el segundo trimestre de 2020?

¿Qué opinión se tiene en las empresas peruanas acerca de los efectos económicos y financieros en las empresas por efecto de la pandemia del COVID19 durante el segundo trimestre de 2020 ?

\subsection{Objetivos de la investigación}

\subsubsection{Objetivo general}

Obtener información que permita conocer el impacto por efecto de la pandemia del COVID-19 en el 2do trimestre de 2020.

\subsubsection{Objetivos específicos (Propósitos específicos mediante los que se puede lograr el objetivo general)}

- $\quad$ Obtener información que permita conocer el estado de la operación comercial de las empresas peruanas durante la pandemia del COVID-19 en el 2do trimestre de 2020.

- $\quad$ Obtener información que permita conocer la opinión sobre los efectos económicos y financieros en las empresas por efecto de la pandemia del COVID-19 durante el 2do trimestre de 2020. 


\subsection{Justificación de la investigación}

\subsubsection{Motivaciones de carácter práctico}

Teniendo bajo contexto que durante la pandemia del COVID-19 se declaró Estado de Emergencia Nacional en Perú, se tiene que el flujo de información empresarial es limitado y resulta en varias ocasiones en desconocimiento y desinformación de la temática empresarial a nivel nacional. La investigación propuesta constituye un medio de contribución para el conocimiento y su consecuente generación de información de la temática empresarial nacional; la investigación propuesta pretende abordar el impacto del COVID19 en la empresa, las ventas, el empleo, las finanzas, expectativa de operación comercial, acceso a programas del gobierno, y la inoperatividad empresarial si es que fuere el caso. Expuesto lo anterior, se deduce que la investigación propuesta genera conocimiento e información de soporte para la toma de decisiones respecto del entorno empresarial peruano.

\subsubsection{Motivaciones de carácter metodológico}

La investigación propuesta se traduce en un medio de aprovechamiento de los datos abiertos del Instituto Nacional de Estadística e Informática (INEI) procedentes de la Encuesta de Opinión sobre el Impacto del COVID-19 en las Empresas que se ejecutó en el mes de julio de 2020 . El aprovechamiento de los datos en cuestión se refiere a su procesamiento para generar información del entorno empresarial peruano durante la pandemia del COVID-19. El aprovechamiento de los datos abiertos del Instituto Nacional de Estadística e Informática (INEI) resulta en una contribución de valor en vista que la encuesta que permitió su recolección tiene objetivos altamente compatibles con los objetivos de la presente investigación; los objetivos son los siguiente de acuerdo a la ficha técnica [5]: (1) Obtener información estadística cualitativa que permita conocer la opinión sobre los efectos económicos y financieros en las empresas por efecto de la pandemia del COVID-19; (2) Elaborar indicadores sobre aspectos cualitativos en los temas relacionados a las ventas, empleo, finanzas, expectativas y accesos a los programas del gobierno, vinculados a los efectos del COVID-19 en las empresas.

\subsubsection{Motivaciones de carácter teórico}

La investigación propuesta permite aportar con proposiciones teóricas respecto de la temática empresarial peruana durante la pandemia del COVID-19 en el 2do trimestre de 2020. La temática empresarial que se aborda refiere al impacto del COVID-19 en la empresa, las ventas, el empleo, las finanzas, expectativa de operación comercial, acceso a programas del gobierno, y la inoperatividad empresarial si es que fuere el caso. El aporte de proposiciones basada en datos resulta de una contribución relevante en cuanto a su calidad de aportadora de objetividad frente a la percepción cualitativa del entorno empresarial peruano que tiene su origen no en datos sino tan solo en eventos relacionados con el avance de la pandemia COVID-19 tanto a nivel nacional como internacional.

En síntesis, la presente investigación aprovecha estadísticamente los datos del Instituto Nacional de Estadística e Informática (INEI) respecto de la encuesta de opinión empresarial peruana sobre el impacto del COVID-19 que obtiene información para conocer la opinión sobre los efectos económicos y financieros en las empresas por efecto de la pandemia del COVID-19 [5]; el aprovechamiento de los datos resulta en el logro de un diagnóstico del estado de operación comercial y opinión sobre los efectos económicos y financieros en las empresas como consecuencia de la pandemia COVID-19.

\section{Marco Teórico}

\subsection{Marco legal nacional}

La investigación propuesta se enmarca legalmente dentro del Decreto Supremo № 044-2020-PCM que declara Estado de Emergencia Nacional por las graves circunstancias que afectan la vida de la Nación a consecuencia del brote del COVID-19 [1]. La investigación propuesta contribuye a mensurar mediante ciertos indicadores los efectos de la promulgación del decreto en cuestión en cuanto que afecta al entorno empresarial peruano por las disposiciones de aislamiento social obligatorio (cuarentena).

Adicionalmente, se considera dentro del marco legal a los decretos que refieren a la reanudación de actividades económicas en forma gradual y progresiva dentro del marco de la declaratoria de Emergencia Sanitaria Nacional por las graves circunstancias que afectan la vida de la Nación a consecuencia del COVID-19; los decretos en mención son el Decreto Supremo $N^{\circ}$ 080-2020PCM, Decreto Supremo No 101-2020-PCM, Decreto Supremo $N^{\circ} 117-2020-\mathrm{PCM}$, Decreto Supremo $\mathrm{N}^{\circ}$ 157-2020-PCM; los decretos en mención refieren a 
la reanudación de actividades en las fases 1 a 4 , respectivamente. La reanudación de actividades se entiende como comenzar la recuperación social y económica incorporando precauciones y medidas de protección necesarias para prevenir los contagios y minimizar el riesgo de un repunte de la enfermedad a causa del COVID-19 [2]. Téngase presente que quien organiza la reanudación de actividades económicas es el Grupo de Trabajo Multisectorial para la reanudación de actividades económicas creado mediante la Resolución Ministerial $N^{\circ}$ 144-2020-EF/15 [6]. La investigación propuesta aporta a las disposiciones de los decretos de reanudación de actividades dado que se difunden opiniones empresariales respecto de las acciones del gobierno peruano; por lo tanto, la investigación se constituye como material informativo con relación a los efectos de las disposiciones gubernamentales respecto de la reanudación de actividades.

El programa "Reactiva Perú" promueve el financiamiento de la reposición de los fondos de capital de trabajo de las empresas que enfrentan pagos y obligaciones de corto plazo con sus trabajadores y proveedores de bienes y servicios, a efectos de asegurar la continuidad en la cadena de pagos en la economía nacional [7]. Además, se cuenta que la implementación del programa "Reactiva Perú" se encuentra regulada en sus aspectos operativos por medio del reglamento operativo del programa "Reactiva Perú" [8]. Expuesto lo anterior, se tiene que la investigación aporta a las disposiciones del Decreto Legislativo $\mathrm{N}^{\circ}$ 1455 y la Resolución Ministerial № 134-2020-EF/15 donde se crea el programa "Reactiva Peru" y se aprueba su respectivo reglamento operativo; el aporte consiste en la provisión del sentimiento empresarial frente a las disposiciones gubernamentales mediante el uso de datos recolectados de la opinión de las empresas respecto de las disposiciones gubernamentales; la recolección de datos se traduce, también, en la provisión de indicadores de opinión empresarial respecto de las disposiciones gubernamentales.

\subsection{Marco nacional}

El marco nacional de la presente investigación queda debidamente contextualizado mediante el Marco Macroeconómico Multianual 2022 - 2025 que provee proyecciones macroeconómicas multianuales oficiales teniendo como base los escenarios nacional e internacional [9]. El Ministerio de Economía y Finanzas [9] afirma que el producto bruto interno $(\mathrm{PBI})$ continuará recuperándose hasta alcanzar los niveles prepandemia del COVID-19 hacia la segunda mitad del año 2021 y durante 2022, lo anterior sería posible gracias al control progresivo de la pandemia del COVID-19 y el avance en el proceso de vacunación, que permitirá incrementar los aforos de las actividades con restricciones. Se dice que las actividades económicas vinculadas a la prestación de servicios, las cuales fueron las más afectadas por la pandemia, retornarían gradualmente a los niveles pre-COVID-19 entre 2023 y 2025 [9]. La investigación propuesta constata y verifica que el impacto de la pandemia del COVID-19 es relativamente negativo para con el entorno empresarial peruano; en buena cuenta la investigación propuesta está alineada con las consideraciones económicas nacionales vigentes.

En el marco de la pandemia del COVID-19, el Ministerio de la Producción [10] divulga como logros de gestión el apoyo al Programa de Apoyo Empresarial (PAE-MYPE) con un fondo de S/ 2000 millones a favor de 156 mil micro y pequeñas empresas (MYPE), y la extensión del periodo de gracia de los créditos de Reactiva Perú y PAEMYPE hasta el 2022. Se piensa que la presente investigación contribuye a la gestión del Ministerio de la Producción en cuanto a facilitar detalles informativos acerca de si las empresas acceden o no a los programas del gobierno, además de los detalles acerca de la opinión empresarial respecto de los programas o medidas de incentivo $\mathrm{y} / \mathrm{o}$ reactivación gubernamentales.

Habiéndose aprobado "El Plan Bicentenario: El Peru hacia el 2021" mediante el Decreto Supremo $N^{\circ}$ 054-2011-PCM donde se aprueba el primer Plan Estratégico de Desarrollo Nacional teniendo como horizonte temporal el año 2021 [11], se tiene que la investigación propuesta pretende contribuir con el plan estratégico peruano en cuanto a insumo de información para el cumplimiento de su función como instrumento evaluador del contexto nacional e internacional y las megatendencias que se perfilan a la fecha. La contribución de la presente investigación se presume ser relevante, debido a que tratando sobre los impactos de la pandemia del COVID-19 y las opiniones empresariales, se aporta a enriquecer la temática de las megatendencias que son objeto del planeamiento estratégico del Perú para lograr una estabilidad macroeconómica y equilibrio; se da por entendido que los impactos de la pandemia COVID-19 constituyen una megatendencia dadas las implicancias tanto a nivel nacional como internacional. 


\subsection{Marco internacional}

En el Marco Macroeconómico Multianual 20222025, el Ministerio de Economía y Finanzas [9] enmarca la economía nacional peruana dentro de una economía global donde se da una recuperación rápida la cual pasaría de contraerse 3.2\% en 2020 a crecer $6.0 \%$ en 2021 . Se tiene también que el bloque de economías emergentes y en desarrollo estarían siendo favorecidas por el mayor crecimiento de China y América Latina dada la mejora de la demanda externa y los altos precios de las materias primas [9]. A nivel internacional se tiene como riesgos los siguientes [9]: (1) la posibilidad de nuevos rebrotes del COVID-19 y la demora en la distribución de las vacunas; (2) deterioro de condiciones financieras; (3) presiones inflacionarias; (4) tensiones geopolíticas y sociales; (5) menor impulso fiscal. Expuesto lo anterior, se afirma que la presente investigación queda contextualizada dentro del escenario internacional propuesto por el Ministerio de Economía y Finanzas (2021) donde se asevera la realidad de una recuperación económica global caracterizada por una menor volatilidad de los mercados financieros, el avance en el proceso de vacunación, y la continuidad de las políticas económicas.

Se afirma que la pandemia del COVID-19 a sumergido a la economía global en la más profunda recesión desde la 2da Guerra Mundial lo que implica que el ingreso per cápita se estima haberse reducido en un $90 \%$ el 2020 [12]. Teniéndose en cuenta que la productividad refiere al Producto Bruto Interno (PBI) por trabajador [12], se afirma que la pandemia constituye un factor adverso que contribuye a la desaceleración de la tasa de crecimiento de la productividad global iniciada después de la Gran Crisis Financiera Global de 2008 [12]. Dada la pandemia del COVID-19, se afirma que las implicancias negativas que conlleva a la productividad son las siguientes [12]: (1) desinversión; (2) Erosión del capital humano y cambios en los mercados laborales; (3) Disminución del impulso en la reasignación laboral; (4) Pesada carga de la deuda. Del mismo modo, Dieppe [12] defiende que hay implicancias positivas de la pandemia del COVID-19 que se constituyen como oportunidades compensatorias para mejorar la productividad tales como las siguientes: (1) Cambios organizacionales y tecnológicos; (2) Implementación de cadenas de suministro diversas y resistentes; (3) Mejoras en la educación; (4) Desarrollo financiero. Expuesto lo anterior, se tiene que la presente investigación queda contextualizada internacionalmente en las apreciaciones de Dieppe [12] sobre la pandemia del COVID-19 como factor adverso a la tasa de crecimiento de la productividad global. Se da por sentado que los efectos de la pandemia del COVID19 han sido bastante similares a nivel internacional. Didier et al. [13] divulgan que la pandemia del COVID-19 ha frenado la actividad económica y se ha dado el fenómeno de la hibernación donde se desacelera la economía hasta tener la pandemia bajo control preservando valiosas y vitales relaciones empresariales para lograr, después, una más rápida recuperación; lo mencionado acerca de la hibernación se traduce en compartir la carga de la inactividad económica entre todas las partes interesadas para la supervivencia empresarial [13]. Del mismo modo, Didier et al. [13] promueve que el financiamiento empresarial extraordinario provisto 0 por los gobiernos 0 entidades financieras de envergadura internacional ayuda a las empresas a cubrir los costos operativos hasta llegar a una situación controlada respecto de la pandemia del COVID-19; el financiamiento en cuestión resultaría importante para no incurrir en problemas de liquides o solvencia [13]. Las apreciaciones mencionadas por Didier et al. [13], acerca del financiamiento empresarial durante la pandemia del COVID-19 como medio para la supervivencia empresarial, se consideran que contextualizan internacionalmente la presente investigación debido a que éstas últimas estarían vinculadas con datos financieros que se usan en la investigación que refieren a la problemática financiera en el Perú durante la pandemia del COVID-19.

\subsection{Aporte como medio de verificación y/o constatación de otros estudios $y / 0$ investigaciones}

Los resultados de la presente investigación aportan a verificar la representatividad de otros estudios $\mathrm{y} / \mathrm{o}$ investigaciones que divulgan acerca de la situación de las empresas peruanas a razón de la pandemia del COVID-19. La Sociedad Nacional de Industrias [4] destaca a abril de 2020 que $59 \%$ de las empresas industriales han paralizado totalmente mientras que un $33 \%$ operan parcialmente; únicamente el $8 \%$ de las empresas industriales se encuentran operando con normalidad. Lo mencionado corresponde a la información provista por 181 empresas [4]. En particular, se piensa que la presente investigación sirve para verificar la representatividad de los resultados provistos por la Sociedad Nacional de Industrias. Además, se piensa que la presente investigación contribuiría a facilitar un panorama más preciso dado que la información con la que se trabaja es provista por 
2,354 empresas de acuerdo a la ficha técnica de encuesta emitida por el Instituto Nacional de Estadística e Informática (INEI) [5].

A nivel internacional ha habido esfuerzos para diagnosticar el estado del mundo empresarial impactado por la pandemia COVID-19. Tal es el caso del trabajo de investigación difundido por Apedo-Amah et al. [14] donde se evalúa el impacto del COVID-19 en el corto plazo en el mundo empresarial a nivel global focalizándose en los países en desarrollo. Habiendo analizado la situación de 100,000 negocios, Apedo-Amah et al. [14] encuentra que la pandemia del COVID-19 resultó en una caída significativa de las ventas, ajustes en el empleo (reducción de horas y despidos), incremento en las restricciones financieras, incremento en el uso de las soluciones digitales, e incertidumbre frente al futuro. La presente investigación contiene hallazgos que permiten constatar que la realidad empresarial peruana es bastante similar a la realidad empresarial internacional como la describe ApedoAmah et al. [14] en cuanto a su situación y respuesta frente al impacto de la pandemia del COVID-19.

Otro esfuerzo internacional que diagnostica la situación empresarial internacional es aquel efectuado por Bachas et al. [15] que utiliza los registros administrativos de impuestos corporativos de 10 países de ingresos bajos y medios con la finalidad de simular cómo la pandemia del COVID19 impacta en las ganancias y el pago de impuestos de las empresas. Bachas et al. (2020) han predicho lo siguiente: (1) menos del $50 \%$ de las empresas permanecería como rentables; (2) se perdería aproximadamente del 5 al $10 \%$ de la nómina anual agregada; (3) se duplicarían las tasas de deserción empresarial (quiebra). Por otro lado, Bachas et al. (2020) afirman que los subsidios de nómina no serían muy útiles para proteger el empleo formal, además, se esperaría un representativo déficit en el ingreso por impuestos de los gobiernos. La presente investigación sirve para constatar que la realidad empresarial peruana es similar al fenómeno empresarial internacional a razón del impacto del COVID-19, en particular, respecto de la rentabilidad empresarial y el comportamiento del empleo como lo exponen Bachas et al. [15].

Otro esfuerzo internacional ha sido llevado a cabo por Cirera et al. [16] donde provee evidencia de 120,000 empresas provenientes de 60 países mayormente en desarrollo respecto de la efectividad de políticas de soporte empresarial por la pandemia del COVID-19. Cirera et al. [16] divulgan que los gobiernos de diferentes países han implementado más de 1600 iniciativas de políticas para dar soporte a pequeñas y medianas empresas. Es de mencionar que un problema del diseño de políticas fue la tenencia de pocos datos y evidencias, sobre todo en países en desarrollo [16]. Cirera et al. [16] concluyen que hay brechas importantes que deben superarse para mejorar el soporte a las políticas de la pandemia del COVID-19 en cuanto al alcance, la focalización, y la eficacia. No obstante, se ha detectado que gran cantidad de empresas se han beneficiado de la asistencia pública provenientes de ciertas políticas que han tenido éxito en mitigar las limitaciones de liquides y reducir los despidos [16]. Por un lado, la presente investigación es un contribuyente de datos y evidencias que se requieren como soporte para la elaboración de políticas; por otro lado, la presente investigación se constituye como un medio para verificar y constatar estudios e investigaciones de envergadura internacional como la investigación de Cirera et al. [16] acerca de las políticas para brindar soporte empresarial durante el impacto de la pandemia del COVID-19.

\section{Método}

\subsection{Formulación y operacionalización de hipótesis}

\subsubsection{Formulación (Las hipótesis están planteadas bajo una forma que permita la verificación)}

$\mathrm{H} 1$ : Las empresas peruanas han presentado una caída en sus ventas durante la pandemia del COVID-19.

H2: Las empresas peruanas han contado con menos trabajadores en la empresa durante la pandemia del COVID-19.

H3: Las empresas peruanas presenta problemas financieros significativos durante la pandemia del COVID-19.

H4: Las empresas peruanas perciben que volverán a sus niveles de venta en entre 6 y 12 meses a partir del mes de julio de 2020.

H5: Las empresas peruanas acceden de manera limitada a los programas de ayuda del Gobierno peruano durante la pandemia del COVID-19.

H6: El nivel de la capacidad instalada operativa incide en la operatividad de la empresa durante el 2do trimestre de 2020. 


\subsubsection{Operacionalización}

Tabla 1: Operacionalización

\begin{tabular}{|c|c|c|c|}
\hline Dimensión & Variable & Indicador & Operacionalización de indicadores \\
\hline Operatividad & $\begin{array}{c}\text { Estado } \\
\text { Operativo }\end{array}$ & Operatividad de la empresa & Estado operativo de la empresa \\
\hline \multirow[t]{2}{*}{ Comercial } & \multirow[t]{2}{*}{$\begin{array}{l}\text { Estado } \\
\text { comercial }\end{array}$} & $\begin{array}{l}\text { Ventas en el segundo trimestre } \\
2020\end{array}$ & $\begin{array}{l}\text { Nivel de ventas de la empresa en el } \\
\text { segundo trimestre } 2020\end{array}$ \\
\hline & & $\begin{array}{l}\text { Capacidad instalada operativa en } \\
\text { el segundo trimestre } 2020\end{array}$ & $\begin{array}{l}\text { Utilización de capacidad instalada } \\
\text { operativa }\end{array}$ \\
\hline Laboral & $\begin{array}{l}\text { Estado } \\
\text { laboral }\end{array}$ & $\begin{array}{l}\text { Fuerza laboral en el segundo } \\
\text { trimestre de } 2020\end{array}$ & $\begin{array}{l}\text { Porcentaje de trabajadores laboró en la } \\
\text { empresa en el segundo trimestre } 2020\end{array}$ \\
\hline Financiera & $\begin{array}{c}\text { Estado } \\
\text { financiero }\end{array}$ & Problemas financieros & Tipo de problemas \\
\hline $\begin{array}{l}\text { Apreciación y } \\
\text { Expectativa }\end{array}$ & $\begin{array}{l}\text { Estado } \\
\text { estratégico }\end{array}$ & Resiliencia & $\begin{array}{l}\text { Tiempo en que la empresa volverá a los } \\
\text { mismos niveles de ventas que tenía } \\
\text { antes del inicio de la pandemia del } \\
\text { COVID-19 }\end{array}$ \\
\hline Gobierno & $\begin{array}{c}\text { Asistencia } \\
\text { gubernamental }\end{array}$ & $\begin{array}{l}\text { Acceso a programas y medidas } \\
\text { de incentivo y/o reactivación del } \\
\text { Gobierno }\end{array}$ & $\begin{array}{l}\text { Tipo de programas y medidas de } \\
\text { incentivo y/o reactivación del Gobierno }\end{array}$ \\
\hline
\end{tabular}

\subsection{Aspectos Metodológicos}

\subsubsection{Tipo de estudio:}

\subsubsection{Descriptivo}

El estudio se considera del tipo descriptivo porque el propósito de la investigación es buscar especificar características las empresas peruanas durante la pandemia del COVID-19; lo anterior resulta útil porque muestra con precisión las dimensiones del comportamiento empresarial peruano durante la pandemia del COVID-19.

\subsubsection{Explicativo}

El estudio se considera del tipo explicativo porque incluye responder a las causas del comportamiento empresarial peruano durante la pandemia del COVID-19 buscando enfocarse en explicarse la razón de ser de este comportamiento; lo anterior resulta útil puesto que proporciona un sentido de entendimiento del comportamiento empresarial peruano durante la pandemia del COVID-19.

\subsubsection{Método de investigación:}

- Método de investigación inductivo

El método que se aplica en la investigación propuesta es el método inductivo donde se analiza una parte de un todo y va desde lo particular a lo general, o de lo individual a lo universal [17]. La presente investigación utiliza los datos de una muestra de 2354 empresas peruanas para diagnosticar y hacer inferencias de la población de empresas en el Perú.

\section{- $\quad$ Método de investigación objetivista}

Se dice que la presente investigación es objetivista puesto que se observa el comportamiento real y tangible para analizarlo y llegar a conclusiones objetivas, además, se tiene por finalidad conocer las características reales del fenómeno observado [17]. En este caso el fenómeno que se observa es el comportamiento de las empresas peruanas durante la pandemia COVID-19.

\section{- Método de investigación estático}

Se dice que la presente investigación procede de un método de investigación estático puesto que sólo se limita a observar los hechos y fenómenos bajo un aspecto concreto y sin admitir ninguna variación en cuanto a alcances, normas y parámetros del comportamiento del propio fenómeno [17]. Siendo el fenómeno observado el comportamiento empresarial peruano durante la pandemia del COVID-19, este se da únicamente durante el 2do trimestre de 2020.

\subsubsection{Fuentes y técnicas de recolección de información}




\section{- $\quad$ Fuentes primarias}

Los datos con los que se trabajan en la presente investigación son procedentes de fuentes primarias, es decir, proceden de las mismas empresas peruanas donde los informantes son el/la propietario/a, gerente/a, contador/a, representante legal o la persona que la empresa designe como tal [5]; se menciona, además, que los datos empresariales del 2do semestre de 2020 han sido recolectados por el Instituto Nacional de Estadística e Informática (INEI) [5].

\section{- $\quad$ Fuentes secundarias}

Se utilizan fuentes secundarias para confrontar los datos de fuentes primarias en la investigación a manera de verificación y constatación del comportamiento empresarial peruano durante la pandemia del COVID-19. Las fuentes secundarias la constituyen datos e información que son provistas por investigaciones $\mathrm{y} / \mathrm{o}$ estudios acerca del comportamiento empresarial durante la pandemia del COVID-19 como los provistos por la Sociedad Nacional de Industrias [4], Apedo-Amah et al. [14], Bachas et al. [15], y Cirera et al. [16].

\subsubsection{Tratamiento de la información}

\subsubsection{Técnicas de análisis estadístico}

Se aplica análisis estadístico descriptivo con la finalidad de describir los datos para cada variable. Se aplica análisis estadístico categórico con la finalidad de estudiar la existencia o no de asociación entre atributos.

\subsection{Impacto que tendrá la investigación en la política pública}

Es evidente que la pandemia del COVID-19 a afectado severamente a las empresas peruanas; no se trata solamente de una caída en las ventas sino, también, se ha reducido el acceso al financiamiento colocando a la empresa en situación de incertidumbre; además, se aprecian problemas de solvencia, desinversión, y pérdida de empleos. En el contexto expuesto, se tiene que las políticas públicas juegan un rol clave para mitigar los impactos negativos de la pandemia del COVID-19, además, de facilitar soporte en la recuperación. El Gobierno Peruano ha introducido programas y medidas de incentivo y/o reactivación; sin embargo, el acceso a estos beneficios es limitado y, en ocasiones, se dan inconvenientes para su acceso en las empresas no tan grandes. Expuesto lo anterior, se piensa que la investigación puede contribuir a la política pública mediante la identificación de empresas que se constituirían como público objetivo para la facilitación de programas y medidas de inventivo y/o reactivación. La identificación de empresas que se pretende es de utilidad porque contiene criterios de diagnóstico que no se utilizan regularmente en la estadística empresarial nacional como son nivel de operatividad de la empresa, accesibilidad a programas de Gobierno para la reanudación de actividades por el brote del COVID-19, entre otros.

\subsection{Limitaciones de la propuesta de investigación}

La presente investigación no ha abarcado la recolección de datos del entorno empresarial peruano; sin embargo, se da por sentado que el proceso de elaboración de la encuesta y la recolección de datos han sido llevados a cabo con efectividad dado que el ejecutor de estas operaciones estadísticas es una entidad confiable, i.e. se trata del Instituto Nacional de Estadística e Informática (INEI).

Del mismo modo, la investigación, únicamente, trabaja con datos que refieren al 2 do trimestre de 2020 respecto del 2 do trimestre de 2019 por lo que la información desarrollada hace referencia, propiamente, no a todo el periodo de la pandemia del COVID-19 sino tan solo al 2do trimestre de 2020. Además, los datos del entorno empresarial peruano se mantienen bajo reserva de acuerdo al Decreto Supremo N 043-2001-PCM, Título Quinto, Capítulo $\mathrm{V}$, sobre el secreto estadístico y la confidencialidad de la información [5].

\section{Resultados}

\subsection{Análisis estadístico descriptivo (Distribución de frecuencias de variables)}

\subsubsection{Estado operativo}


Estado de la empresa como consecuencia del COVID-19 (P_4_1)

2do trimestre de 2020

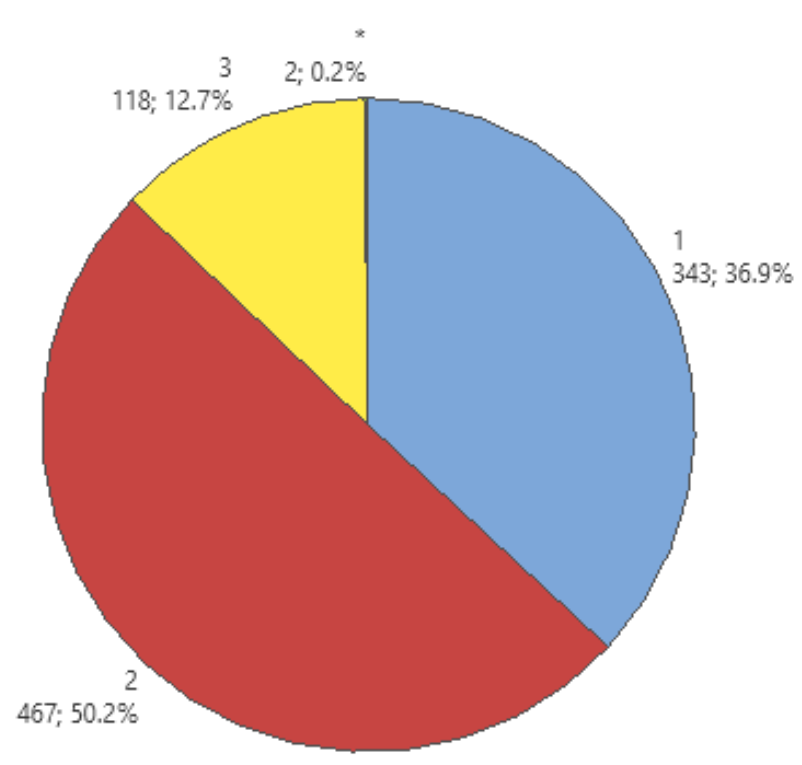

Categoría

Operativa

Parcialmente Operativa

Inoperativa

*

Figura 1. Estado operativo de las empresas durante el 2do trimestre de 2020

\subsubsection{Estado comercial}

El nivel de ventas de la empresa en el 2do trimestre 2020 con respecto al 2 do trimestre de 2019 Base 930 empresas

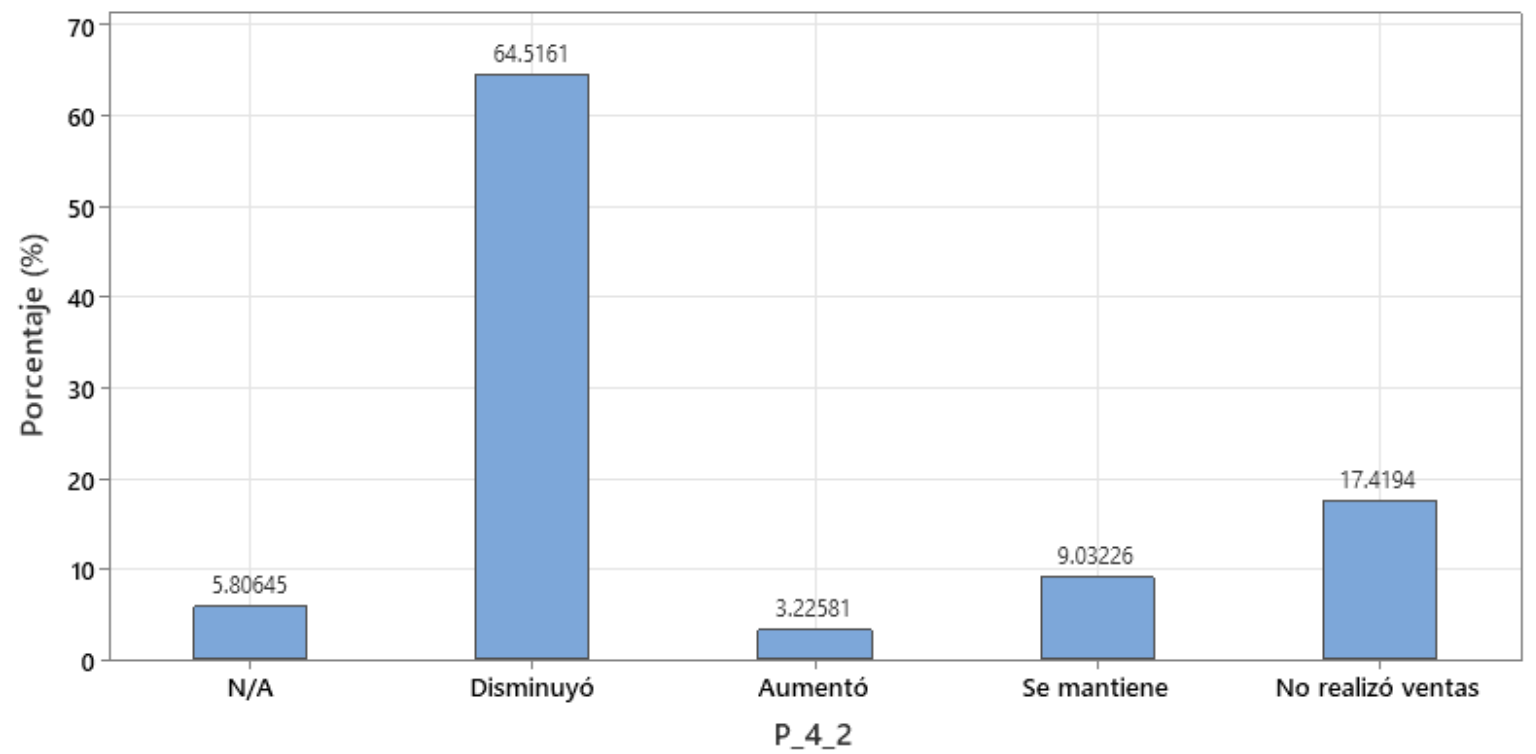

Percent is calculated within all data.

Figura 2. Nivel de ventas durante el 2do trimestre de 2020 
Nivel de capacidad instalada operativa que utilizó la empresa 2do trimestre de 2020

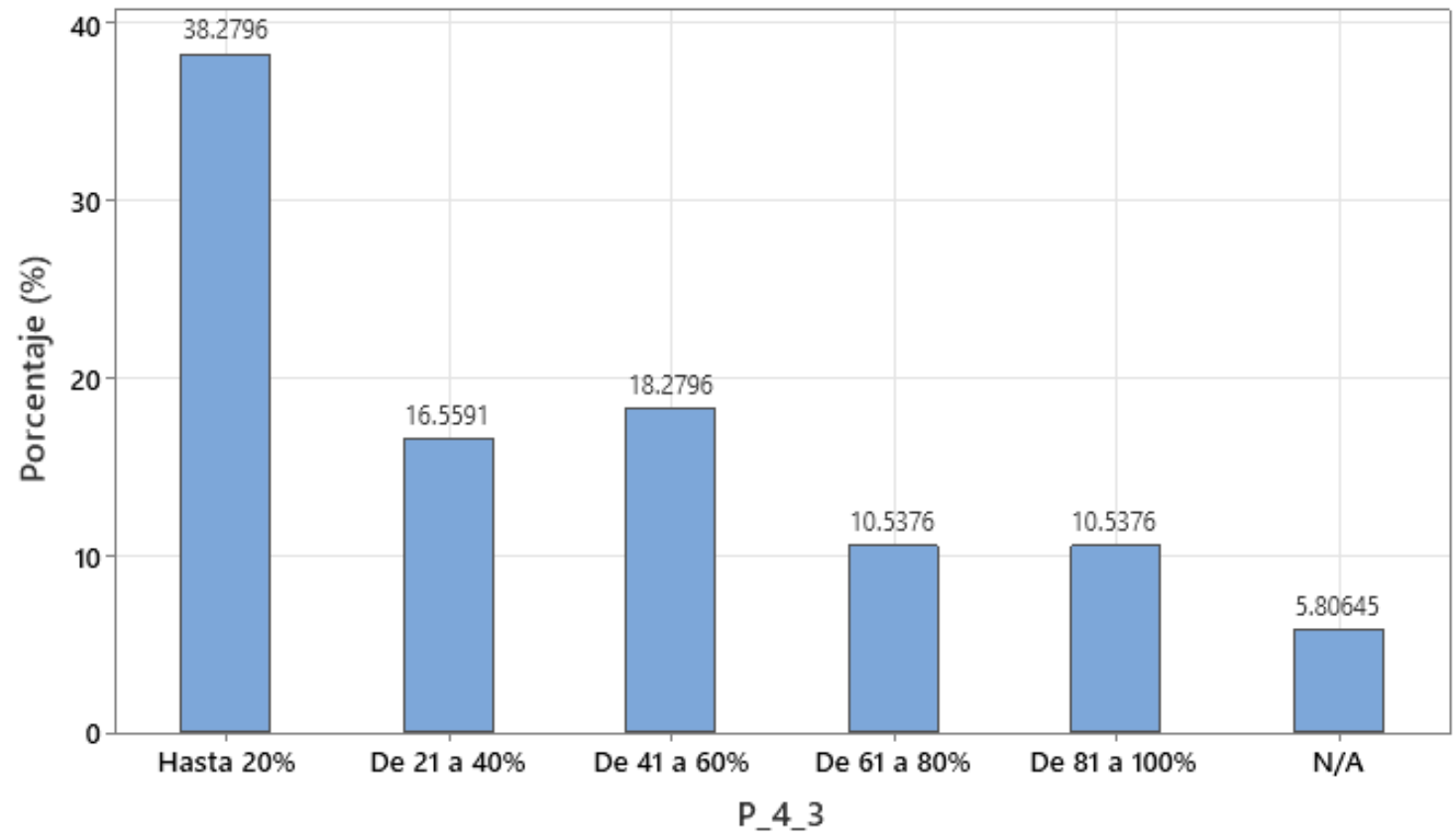

Percent is calculated within all data.

Figura 3. Nivel de capacidad instalada operativa durante el 2do trimestre de 2020

\subsubsection{Estado laboral}

Porcentaje de trabajadores que laboró en la empresa 2do trimeste 2020

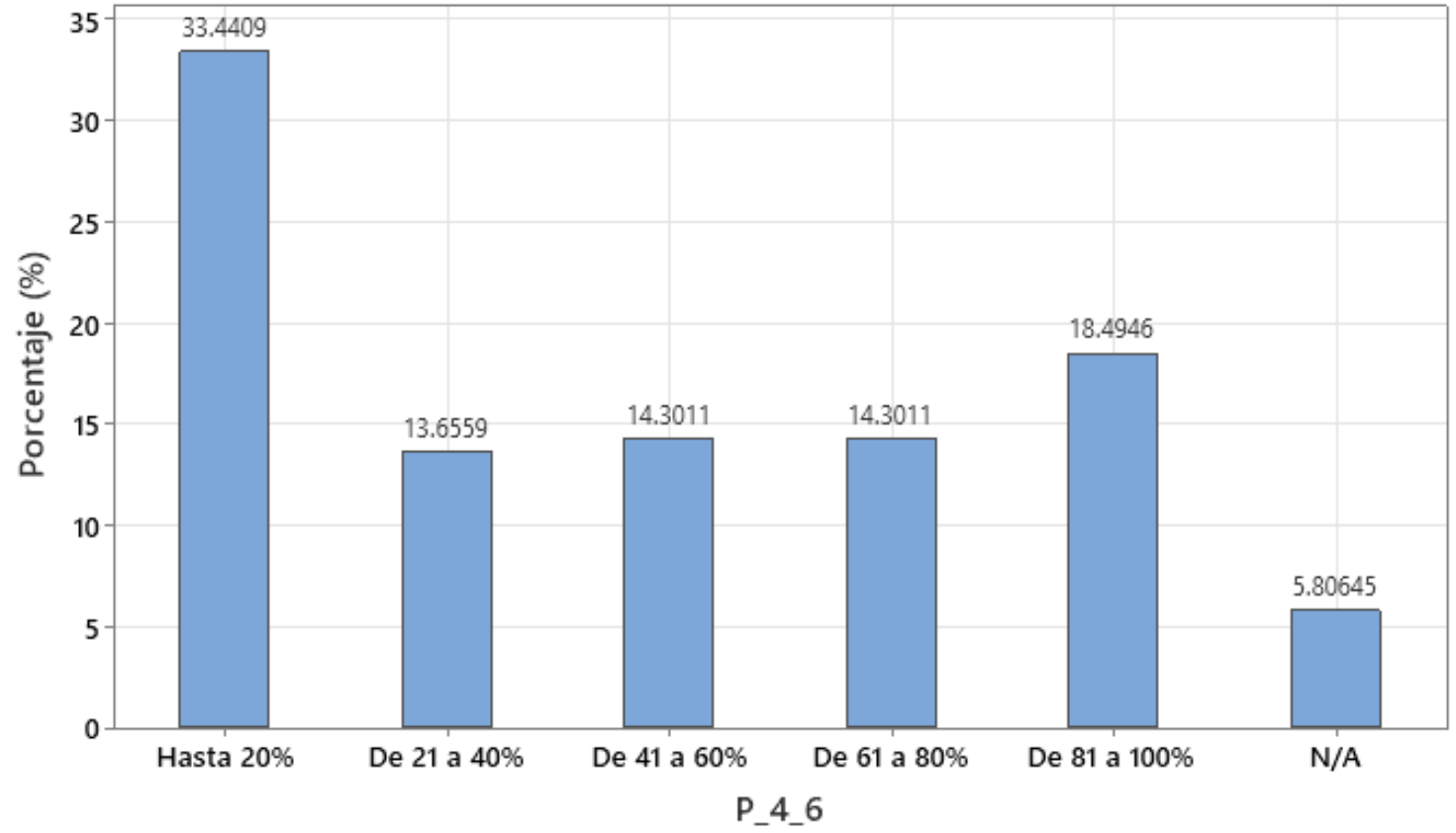

Percent is calculated within all data.

Figura 4. Porcentaje de trabajadores que laboró en la empresa en el 2do trimestre de 2020 


\subsubsection{Estado financiero}

\section{Problemas financieros durante pandemia} 2do trimestre de 2020

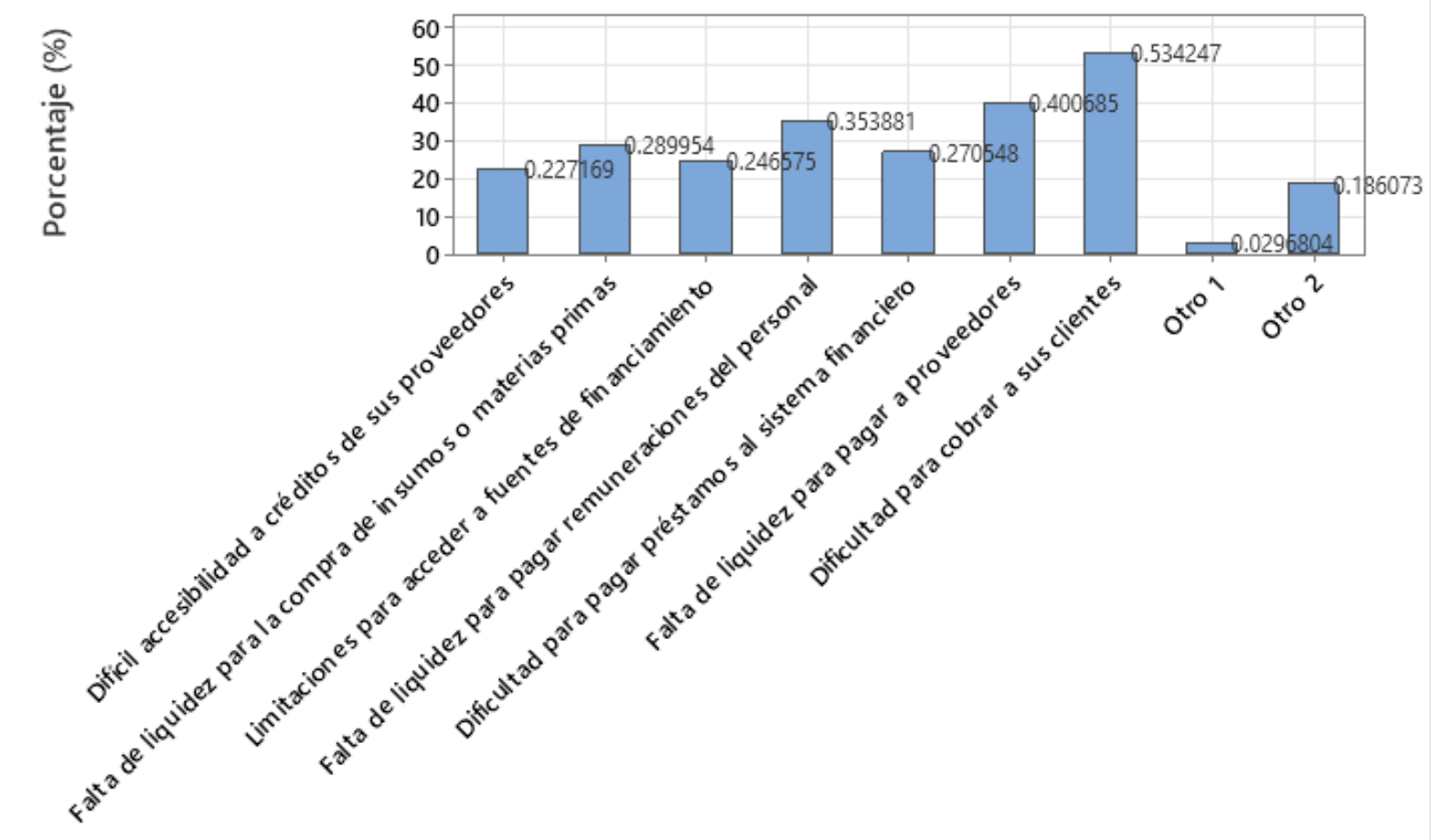

Figura 5. Problemas financieros durante la pandemia en el 2 do trimestre de 2020

\subsubsection{Estado estratégico}

Estimación en meses acerca del retorno a los niveles de ventas antes de la pandemia

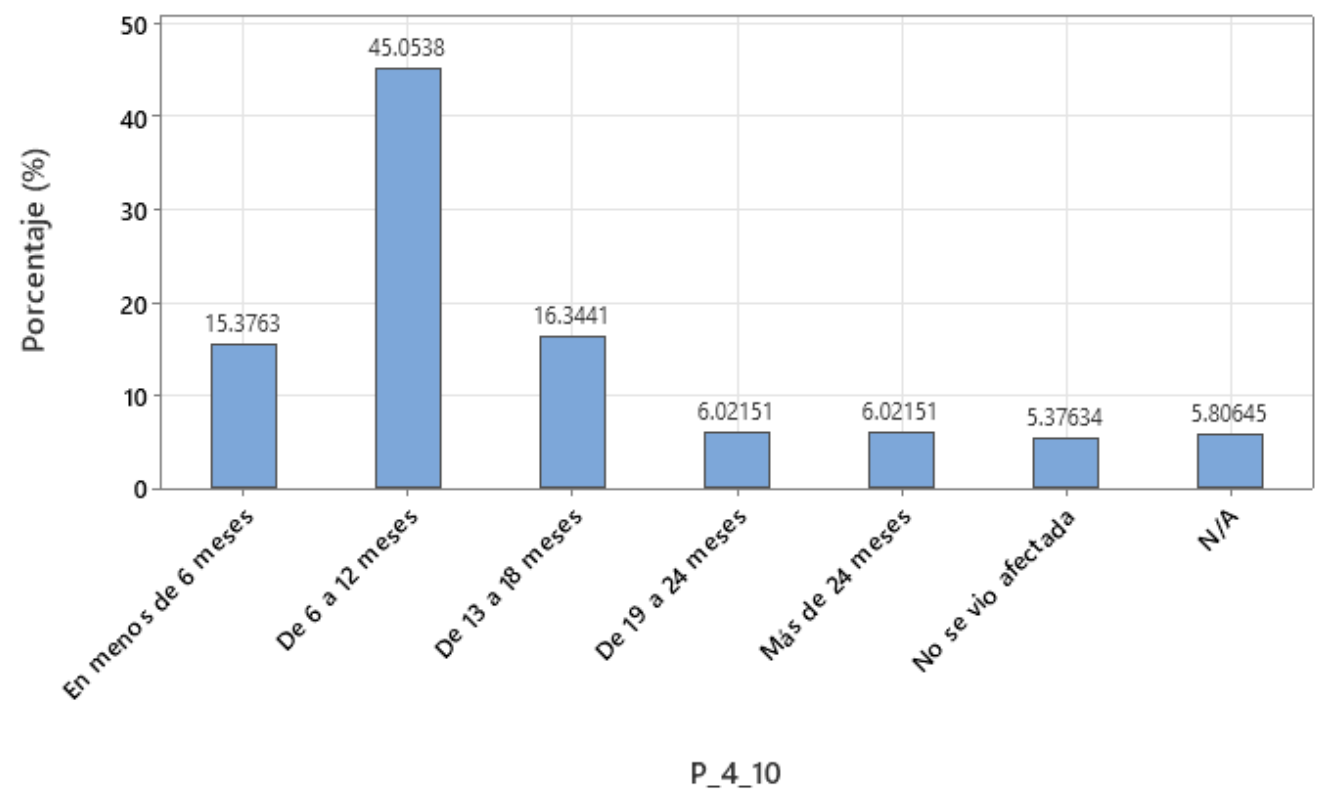

Percent is calculated within all data.

Figura 6. Expectativa de retorno a los niveles de venta prepandemia en el 2 do trimestre de 2020 


\subsubsection{Asistencia gubernamental}

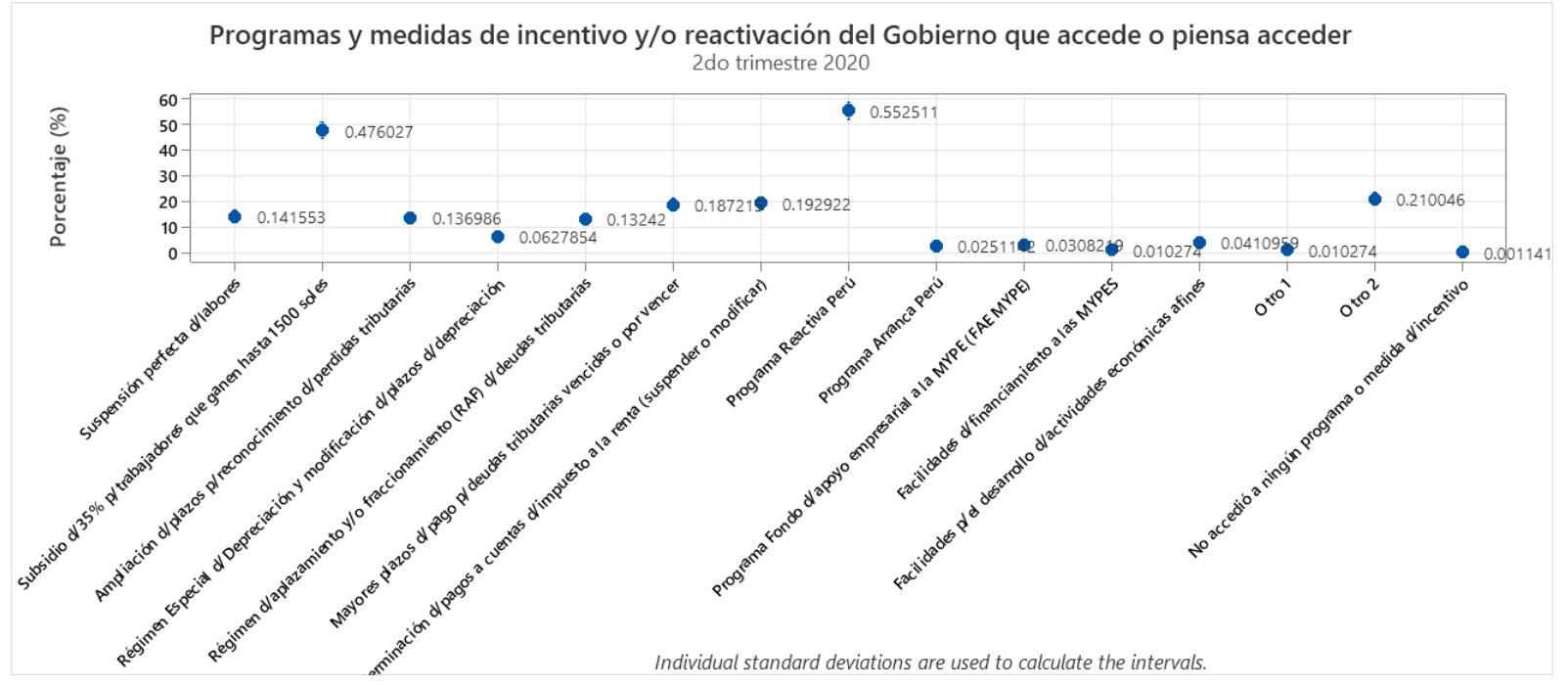

Figura 7. Programas y medidas de incentivo y/o reactivación del Gobierno que accede o piensa acceder en el 2do trimestre de 2020

\subsection{Evaluación de la confiabilidad, validez, y objetividad}

\subsubsection{Confiabilidad}

Se aplica la medida de coherencia o consistencia interna para estimar la confiabilidad; se utiliza el coeficiente Alfa de Cronbach para estimar la confiabilidad. El estadístico de fiabilidad se computó en 0.3643 lo cual se evalúa como muy baja.

\subsubsection{Validez}

La validez suficiente del contenido se da por sentada debido a que el instrumento ha sido aplicado y construido por el Instituto Nacional de Estadística e Informática (INEI) lo cual asegura que las dimensiones medidas por el instrumento son representativas del universo. Únicamente es observable que el instrumento podría carecer de una suficiente representatividad nacional dado que la muestra de empresas peruanas procede no de todos los departamentos del país más solo de Lima, Callao, Arequipa, Piura y Trujillo [5].

\subsubsection{Objetividad}

La objetividad del instrumento es aceptable debido a que el instrumento es suficientemente permeable a la influencia de sesgos y tendencias de los investigadores que lo administran, califican e interpretan. El instrumento se encuentra a disposición del público en general con suficiente información para su utilización en la página web oficial del Instituto Nacional de Estadística e Informática (INEI).

\subsection{Análisis estadístico de datos categóricos}

Se lleva a cabo un análisis de datos categóricos para con las variables operatividad de la empresa (P_4_1) y la variable nivel de la capacidad instalada ( $\left.\mathrm{P}_{-} 4 \_3\right)$. Los resultados estadísticos figuran a continuación. 


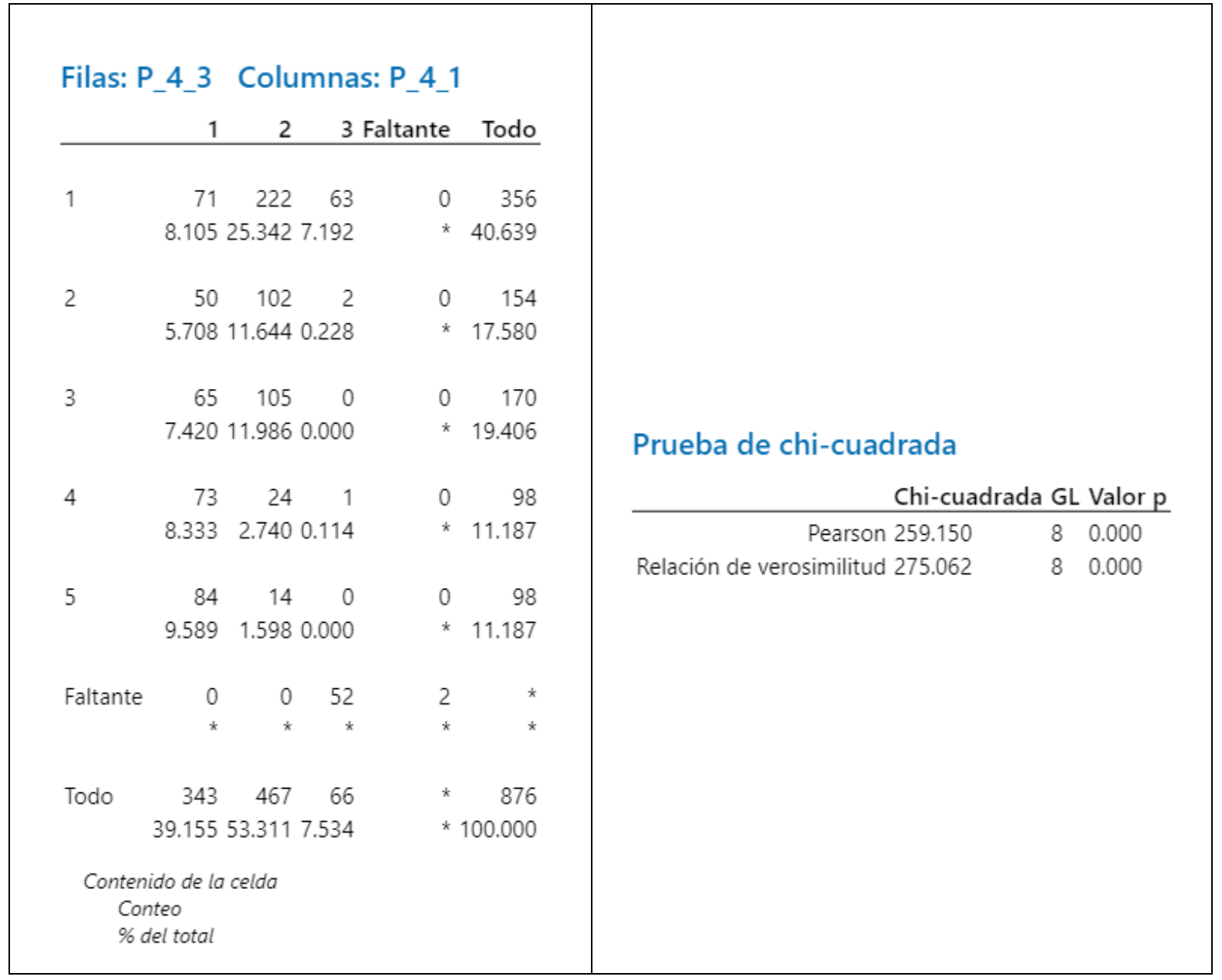

Figura 8. Resultados del análisis de datos categóricos (Tabulación cruzada y Chi-cuadrada)

Nota1. Los valores 1, 2, y 3 de las columnas que pertenecen a la variable P_4_1 corresponden a la operatividad de la empresa y refieren a "operativa", "parcialmente operativa", y "inoperativa”, respectivamente (véase la figura 1).

Nota2. Los valores 1, 2, 3, 4, y 5 de las columnas que pertenecen a la variable $\mathrm{P}$ 4_3 corresponden al nivel de capacidad instalada operatividad y refieren a "hasta $20 \%$ ", "de $21 \%$ a $40 \%$ ", "de $41 \%$ a $60 \%$ ", "de $61 \%$ a $80 \%$ ", y " $81 \%$ a $100 \%$ ", respectivamente (véase la figura 3 ).

Nota3. Los resultados en la figura 8 indican, particularmente, que la operatividad empresarial y el nivel de la capacidad instalada empresarial son variables que tienen asociación puesto que los valores $p$ de la prueba de Pearson y de la prueba de relación de verosimilitud son menores a 0.05 .

\section{Discusión y Conclusiones}

empresas encuestadas (véase la figura 2). No obstante, se estima mayormente que las empresas

\subsection{De los resultados}

La investigación constata que verdaderamente el impacto de la pandemia del COVID-19 ha sido significativamente negativo en las empresas peruanas donde se evidencian aproximadamente un $62.9 \%$ de empresas encuestadas o parcialmente operativas o inoperativas durante el 2do trimestre de 2020 (véase la figura 1). Al panorama anterior, se suma la reportación de que el nivel de ventas en la empresas disminuyó para con un $64.5 \%$ de las peruanas retornen a sus niveles de venta anteriores a la pandemia en un plazo de 6 a 12 meses pasado el 2do trimestre de 2020 (véase la figura 6).

Del mismo modo, se aprecia el impacto negativo de la pandemia en las empresas peruanas respecto del nivel de capacidad instalada operativa utilizada en el 2 do trimestre de 2020 donde cerca del $73.2 \%$ de empresas encuestadas han utilizada su capacidad instalada operativa hasta el $60 \%$ (véase la figura 3). 
Adicionalmente, se demuestra que hay asociación entre el nivel de capacidad instalada operativa y la operatividad de la empresa (véase la figura 8).

El impacto negativo de la pandemia se evidencia también en el porcentaje de trabajadores que labora durante el 2 do trimestre de 2020 donde se tiene que aproximadamente el $61.4 \%$ de las empresas encuestadas operaban hasta con $60 \%$ de los trabajadores (véase la figura 4).

La pandemia del COVID-19 ocasionó una serie de problemas financieros durante el 2 do trimestre de 2020. Se tiene que los problemas más representativos que enfrentaron las empresas peruanas durante el 2do trimestre de 2020 de acuerdo a la encuesta de referencia fueron la dificultad para cobrar a sus clientes, la falta de liquidez para cobrar a sus clientes, y la falta de liquidez para pagar remuneración al personal (véase la figura 5).

La asistencia gubernamental se considera importante para las empresas peruanas durante el 2do trimestre de 2020. Las empresas encuestadas han accedido o piensan acceder mayormente al Programa Reactiva Perú y al subsidio del $35 \%$ para los trabajadores que ganen hasta 1500 soles (véase la figura 7).

\subsection{Comprobación de hipótesis}

La hipótesis $1(\mathrm{H} 1)$ se acepta como tendencia puesto que la mayor parte de empresas peruanas encuestadas o no realizaron ventas o en su defecto tuvieron disminución de estas en $17.41 \%$ y $64.5 \%$, respectivamente (véase la figura 3). La hipótesis 2 $(\mathrm{H} 2)$ se acepta como tendencia significativa puesto que las empresas peruanas encuestadas reportan mayormente (i.e. en un $61.4 \%$ ) hasta $60 \%$ de trabajadores que laboraron en las empresas (véase la figura 4). La hipótesis 3 (H3) se acepta como tendencia puesto que las empresas peruanas encuestadas reportan como principales problemas financieros (véase figura 5): (1) dificulta para cobrar a sus clientes; (2) Falta de liquidez para pagar a proveedores. La hipótesis $4(\mathrm{H} 4)$ se acepta como tendencia porque las empresas peruanas encuestadas reportan de 6 a 12 meses como expectativa de retorno a los niveles de ventas antes de la pandemia (véase la figura 6). La hipótesis 5 (H5) se acepta como tendencia puesto que las empresas peruanas encuestadas reportan acceder o pensar en acceder con poca frecuencia a los programas y medidas de incentivo y/o reactivación del gobierno (véase la figura 7). La hipótesis $6(\mathrm{H} 6)$ se acepta como tendencia empresarial en cuanto que hay asociación entre el nivel de la capacidad instalada operativa y la operatividad de la empresa (véase la figura 8).

\subsection{Conclusiones}

La presente investigación constituye un medio informativo donde se constata que el impacto de la pandemia del COVID-19 ha sido negativo para las empresas peruanas durante el 2do trimestre de 2020. El impacto negativo de las empresas peruanas durante el 2do trimestre de 2020 se evidencia a razón de la inoperatividad u operatividad parcial de las empresas, la disminución de ventas, el funcionamiento con un nivel de capacidad instalada menor al regular, la tenencia de menor cantidad de trabajadores, la tenencia de problemas financieros, y la poca accesibilidad a la asistencia gubernamental para la reactivación. Lo expuesto anteriormente hace que la realidad del entorno empresarial peruano sea acorde con el entorno internacional donde también se verifica un impacto negativo en el entorno empresarial.

La presente investigación contiene hallazgos acerca de la percepción del entorno empresarial peruano acerca de los efectos económicos y financieros por efecto de la pandemia del COVID-19; lo anterior se evidencia en que se tiene como tendencia en el entorno empresarial peruano que se espere mayormente que el nivel de ventas retorne a los niveles antes de la pandemia dentro de 6 a 12 meses a partir del 2do trimestre de 2020.

\section{Agradecimientos}

Se agradece a la institución nacional peruana Instituto Nacional de Estadística e Informática (INEI) por su labor de poner a disposición datos, del público en general, datos estadísticos de interés nacional.

\section{Referencias}

[1] Presidencia del Consejo de Ministros. (2020, 15 de marzo). Decreto Supremo $N^{\circ}$ 044-2020-PCM, Decreto Supremo que declara Estado de Emergencia Nacional por las graves circunstancias que afectan la vida de la Nación a consecuencia del brote del COVID-19. [En línea]. Disponible: https://www.gob.pe/institucion/pcm/ normas-legales/460472-044-2020-pcm

[2] Presidencia del Consejo de Ministros. (2020, 2 de mayo). Decreto Supremo $\mathrm{N}^{\circ}$ 080-2020-PCM, Decreto Supremo que aprueba la reanudación de actividades económicas en forma gradual y progresiva 
dentro del marco de la declaratoria de Emergencia Sanitaria Nacional por las graves circunstancias que afectan la vida de la Nación a consecuencia del COVID19. [En línea]. Disponible: https://www.gob.pe/institucion/pcm/ normas-legales/544911-080-2020-pcm

[3] Dirección de Promoción Minera. 2020 Anuario Minero / Reporte Estadístico. Lima, Perú: Ministerio de Energía y Minas, 2021.

[4] Sociedad Nacional de Industrias, Resultados Encuesta COVID-19: Situación de las Empresas Industriales en el Perú. Lima, Perú: Sociedad Nacional de Industrias (SNI), 2020.

[5] Instituto Nacional de Estadística e Informática (INEI). Encuesta de Opinión sobre el Impacto del COVID-19 en las Empresas - Ficha Técnica. Lima, Perú: Instituto Nacional de Estadística e Informática (INEI), 2020.

[6] Ministerio de Economía y Finanzas. (2020, 25 de abril). Resolución Ministerial № 1442020-EF/15, Conforman Grupo de Trabajo Multisectorial para la reanudación de las actividades económicas. [En línea]. Disponible:

https://www.gob.pe/institucion/mef/ normas-legales/484405-144-2020-ef-15

[7] Decretos Legislativos. (2020, 6 de abril). Decreto Legislativo $N^{\circ}$ 1455, Decreto Legislativo que crea el programa "Reactiva Perú" para asegurar la continuidad en la cadena de pagos ante el impacto del COVID-19. [En línea]. Disponible: https://www.gob.pe/institucion/

presidencia/normas-legales/580222-1455

[8] Ministerio de Economía y Finanzas. (2020, 13 de abril). Resolución Ministerial No 1342020-EF/50, Aprueban el Reglamento Operativo del Programa "REACTIVA PERÚ". [En línea]. Disponible: https://www.gob.pe/institucion/mef/normaslegales/473523-134-2020-ef-50

[9] Ministerio de Economía y Finanzas. Marco Macroeconómico Anual 2022-2025. Lima, Perú: Ministerio de Economía y Finanzas, 2021.

[10] Ministerio de la Producción (PRODUCE). Gobierno de Transición y Emergencia (noviembre 2020 - julio 2021). Lima, Perú, 2021. [En línea]. Disponible: https://www.gob.pe/institucion/produce/ informes-publicaciones/2041620-gobiernode-transicion-y-emergencia-noviembre2020-julio-2021
[11] Centro Nacional de Planeamiento Estratégico (CEPLAN). Plan Bicentenario: El Perú hacia el 2021. Lima, Perú: Centro Nacional de Planeamiento Estratégico (CEPLAN), 2011.

[12] A. Dieppe. Global Productivity / Trends, Drivers and Policies [Productividad global | Tendencias, impulsores y políticas]. Washington, D.C., United States: World Bank Group, 2021.

[13] T. Didier, F. Huneeus, M. Larrain, y S. L: Schmukler. Financing Firms in Hibernation During the COVID-19 Pandemic. Research and Policy Briefs, no. 30 [Financiamiento de empresas en hibernación durante la pandemia de COVID-19. Resúmenes de investigación y políticas, no. 30]. Washington, DC., United States: World Bank, 2020.

[14] M-C- Apedo-Amah, B. Avdiu, X. Cirera, M. Cruz, E. Davies, A. Grover, . . . T. Tran. Unmasking the Impact of COVID-19 on Businesses: Firm Level Evidence from Across the World. Policy Research Working Paper; No. 9434 [Desenmascarando el impacto de COVID-19 en las empresas: evidencia a nivel de firmas de todo el mundo. Documento de trabajo de investigación sobre políticas; № 9434]. Washington, DC., United States: World Bank, 2020.

[15] P. Bachas, A. Brockmeyer, \& C. Semelet. The Impact of COVID-19 on Formal Firms: Micro Tax Data Simulations across Countries [EI impacto de COVID-19 en las empresas formales: simulaciones de microdatos de impuestos entre países]. Washington, DC., United States: World Bank, 2020.

[16] X. Cirera, M. Cruz, E. Davies, A. Grover, L. lacovone, J. E. L. Cordova, . . . J. Torres. Policies to Support Businesses through the COVID-19 Shock : A Firm-Level Perspective. Policy Research Working Paper;No. 9506 [Políticas para apoyar a las empresas a través del impacto de COVID19: una perspectiva a nivel de empresa. Documento de trabajo de investigación sobre políticas; núm. 9506]. Washington, DC., United States: World Bank, 2021.

[17] C. Muñoz. Cómo elaborar y asesorar una investigación de tesis (2da ed.). Naucalpan de Juárez, México: Pearson Educación de México, S.A. de C.V., 2011. 